\title{
Macpherson Prize
}

The Macpherson Prize, established in honour of Crawford Brough Macpherson (1911-1987), internationally renowned political theorist, will be awarded in 1998. The prize, to which is attached a monetary award in the amount of $\$ 750$, is awarded biennially to the author of the best book published in English or French in political theory in the preceding two-year period. The award-winning book must be singleauthored and will be selected by the Board of Directors of the Canadian Political Science Association, upon the recommendation of the Prize Jury. No edited texts, textbooks or collections of essays will be considered. The author must be either a Canadian citizen or a permanent resident (landed immigrant) who resides in Canada. The Macpherson Prize is sponsored by the University of Toronto Press Inc. Individuals who have published a book in 1996 or 1997 which is eligible for the prize should ensure that their publishers forward one copy of the book by December 10, 1997, to each of: (1) Macpherson Prize Jury, Canadian Political Science Association, Suite 205, 1 Stewart Street, Ottawa, Ontario K1N 6H7; (2) Macpherson Prize Jury, Professor Will Kymlicka, Department of Philosophy, University of Ottawa, 70 Laurier Avenue, 2nd Floor, Ottawa, Ontario K1N 6N5; (3) Macpherson Prize Jury, Monsieur Michel Duquette, Département de science politique, Université de Montréal, C.P. 6128, Succursale «A», Montréal, Québec H3C 3J7; and (4) Macpherson Prize Jury, Professor Katherine Fierlbeck, Department of Political Science, Dalhousie University, Halifax, Nova Scotia B3H 4H6.

\section{Prix Macpherson}

Le Prix Macpherson a été créé en hommage à Crawford Brough Macpherson (1911-1987), grand spécialiste de la théorie politique dont la réputation dépassait nos frontières. L'Association décernera le Prix Macpherson, d'une valeur de 750 \$, en 1998 à l'auteur ou l'auteure du meilleur livre publié en français ou en anglais sur un sujet traitant de la théorie politique au cours de la période correspondante de deux ans. Le Conseil d'administration de l'Association canadienne de science politique désignera le lauréat ou la lauréate à la suite d'une recommandation du Jury du Prix Macpherson. Les manuels scolaires, les livres publiés sous la direction d'une personne ou d'une équipe et les collec- 